\title{
Enterprise engineering methods and tools which facilitate simulation, emulation and enactment via formal models
}

\author{
R.H. Weston and P.J. Gilders \\ MSI Research Institute, \\ Dept. of Manufacturing Engineering, \\ Loughborough University of Technology, \\ Loughborough, Leics, UK. \\ Tel: +44 1509 222907, Fax: +44 1509267725 \\ R.H.Weston@lut.ac.uk,P.J.Gilders@lut.ac.uk
}

\begin{abstract}
In the current industrial environment of increasing competition in a globalised marketplace, businesses must strive to closely satisfy ever changing customer desires. Correspondingly, manufacturing systems need be more responsive to the evolution of business requirements and ensure that they directly support these changing demands. Enterprise modelling has contributed significantly to achieving these goals by providing a greater level of consistency between high level requirements definition and lower level system design. However, in order to be truly useful to businesses, enterprise models must be of sufficient detail and provide adequate scope to enable the models to be translated into workable business solutions. Additionally, the variety of available enterprise modelling methods and tools need to be positioned by a common framework, such that relationships between different modelling methods can be identified.

This paper introduces the work carried out at the MSI Research Institute at Loughborough University which aims to provide a toolset to support the lifecycle of enterprise modelling and to support model enactment through the provision of runtime control, resource allocation and information management. Additionally, the paper provides an example application of part of the toolset and concludes by positioning the toolset within the GERAM framework for enterprise modelling.
\end{abstract}

\section{Keywords}

Enterprise, lifecycle, formal, systems, integration, manufacturing, modelling 


\section{INTRODUCTION}

Much of the current literature on manufacturing enterprises is centred on problems and solutions related to the realisation of customer-driven products and services, this in the face of global competition. More than ever before manufacturing enterprises must be highly responsive to changing needs, constantly reforming themselves so that the activities they carry out remain closely aligned to well defined business goals. To remain competitive, each enterprise is required to maximise the benefits arising from its core competences and where necessary negotiate and form alliances on a global scale with other enterprises that have complementary capabilities (Weston 1993).

The problem of aligning enterprise activities with business goals is non-trivial and implies the need to realise business integration as depicted by Figure 1. Indeed to achieve such an

Figure 1. Need for a consolidated life-cycle engineering approach which handles business, application and physical integration

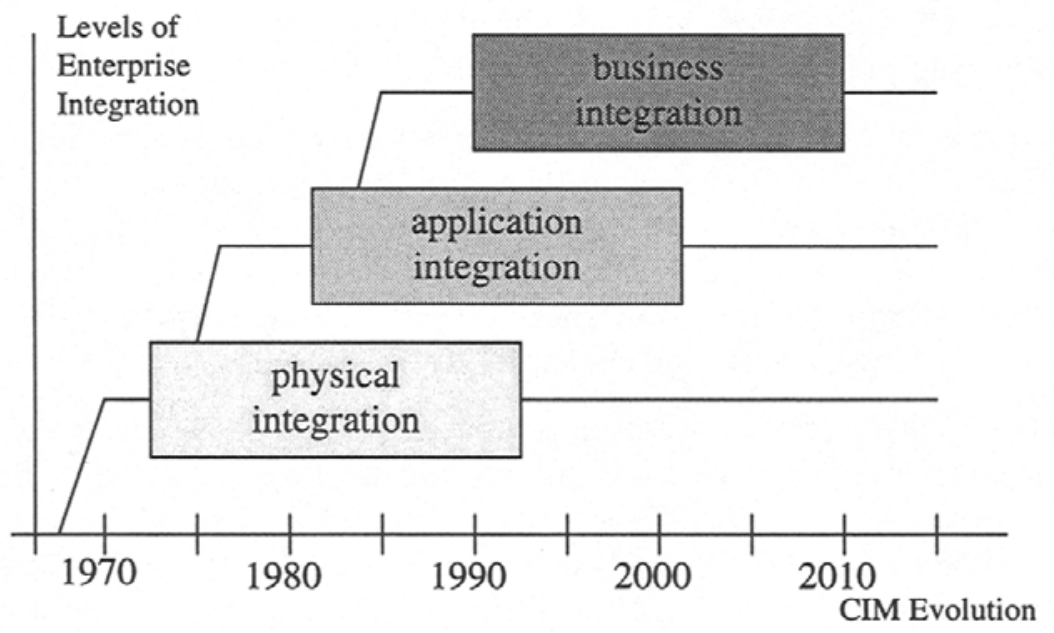

alignment, relatively abstract definitions of business requirements need to be mapped onto more completely defined specifications of the interworking of software embedded in peoplecentred and machine systems; which in turn need to be mapped definitively into physical activities (performed with a particular set of resources) and their associated work and information flows. This paper outlines new methods and techniques which support this need, which were conceived and developed at the MSI (Manufacturing Systems Integration) Research Institute at Loughborough University and implemented in the form of methods workbenches which build on, extend and consolidate research outputs of the enterprise integration community world-wide.

The methods and techniques devised at MSI focus on making enterprise modelling usable, this in a manner which semi-automates the rapid prototyping of model-driven integrated manufacturing systems. Indeed by using a consistent set of models in support of the lifephases of enterprises it has proved possible to produce more effective and reconfigurable solutions with reduced engineering effort and leadtimes. 


\section{SUPPORTING AND USING ENTERPRISE MODELS}

The extremely high levels of complexity inherent in a typical manufacturing enterprise necessitate decomposition and modelling and the use of enterprise models so that solutions can be defined and evolved. Enterprises need to be described from a variety of perspectives (or viewpoints) and at different levels of abstraction (i.e. scope and completeness) before they can be characterised in a meaningful way and the resulting models can become sufficiently complete and accurate for them to be used effectively. Also consistency between the various models needs to be maintained (i.e. between these perspectives and abstraction levels), this requiring the definition and use of an appropriate modelling framework or architecture. Furthermore, as the characteristics of an enterprise change so must attributes of the enterprise models used to describe them. Indeed enterprise models should help support the various phases of the life-cycle of enterprises.

Enterprise Models will typically contain both specific knowledge (about a given enterprise) and generic knowledge (applicable to a class of enterprise or enterprise component). The generic knowledge (Bernus 1995) may take the form of ontological theories (generally applicable rules and facts) and reusable enterprise models (capturing some common part of an enterprise).

It is important also to appreciate that models themselves will be living things and as such will themselves have a life-cycle. As explained later in this paper, enterprise models can have a variety of uses and associated modelling methodologies are typically required to support each use. However, such methodologies should support model capture (i.e. formulation of the models), validation (in terms of the completeness, consistency and correctness of models) and maintenance (i.e. the change, release and enhancement of models).

It is also import to distinguish between methodologies defined to structure and support the life-cycle of an enterprise (i.e. enterprise engineering methodologies) and those used to structure and support the life-cycle of models (i.e. modelling methodologies). Modelling methodologies and their underlying models and modelling languages will typically be more general purpose in nature and as such are less likely to be tied to specific characteristics of a type of enterprise, albeit that they might be best suited for modelling from a particular perspective, level of abstraction or in support of a given life-phase. Conversely enterprise engineering methodologies will typically be linked to use of one or more modelling methodologies and often will be geared to supporting a particular type of enterprise. Hence, known characteristics (i.e. knowledge) about an enterprise type will be embedded within an enterprise engineering methodology; this implying that it can be more readily applied but that a range of such methods will be needed to cover all types of enterprise.

Enterprise models which take a variety of forms have been produced and used by both business and technical communities. Hence it is widely understood by researchers in these fields that it will be necessary to resolve differences between these forms and communities before business integration (i.e. close alignment between enterprise activities and business goals) can be readily achieved.

Commonly researchers and practitioners within the business community have focused on deriving abstract holistic models of enterprises with the prime use being to gain a better understanding of such systems and associated competitive scenarios. Their models may 
include formal descriptions but seldom will it be possible to formally define complete descriptions of processes and systems which include people adopting highly individualistic and innovative roles.

Conversely, researchers within the technical community normally focus more definitively on a given perspective of an enterprise, often with a narrower scope but seeking where possible for more complete and formal descriptions. More complete models can be used with great benefit in a variety of ways. Not only may they be used to gain a better conceptual understanding of systems and possible solutions but they can also be used to structure and semi-automate the creation of those solutions and be embedded within them to help control and maintain them during their operation.

Bearing in mind the various requirements and conditions identified above, the enterprise integration community have made significant advances in defining first generation architectural frameworks, enterprise engineering methodologies, modelling methodologies, enterprise models, ontologies and infrastructural elements. As part of this activity the IFAC/ IFIP Task Force have sought to define GERAM (a Generic Enterprise Reference Architecture and Methodology) which seeks to unify definitions used by researchers world-wide in respect of methods, models and tools needed to build the integrated enterprise (Bernus 1995). GERAM is generic because it applies to most, potentially all types of enterprise. The coverage of the framework spans products, enterprises, enterprise integration and strategic enterprise management, with the emphasis being on the middle two. Currently GERAM is being developed by consolidating aspects of previously analysed architectures, such as the Purdue Enterprise Reference Architecture (Williams 1992), the GRAI Integrated Methodology (Vallespur 1991), CIM-OSA (Vernadat 1992) and TOVE (Fox 1992).

The remainder of this paper will describe on-going research at the MSI Research Institute at Loughborough University which seeks to provide modelling technology and enterprise engineering tools in support of the development and use of GERAM.

\section{THE MODEL DRIVEN CIM PROJECT}

Over the last four years, MSI research within a UK EPSRC funded project known as ModelDriven CIM, has investigated means of making enterprise modelling technology more usable. Here the aim has been to deliver the modelling technology so that it can:

- structure and automate (or semi-automate) life-cycle engineering processes involved in creating integrated manufacturing systems;

- realise solutions in which enterprise activities are driven by models, which closely reflect business objectives;

- realise solutions rapidly (and with reduced engineering effort and cost) so that more responsive enterprises are produced;

- generally maintain, enhance and make available models (and hence knowledge) describing enterprises.

Further requirements of the project were to demonstrate the concepts by building example solutions and to create methods workbenches and thereby facilitate industrial and commercial 
exploitation.

Prior to 1990, MSI research led to the CIM-BIOSYS Integrating Infrastructure and its industrial application in building a variety of integrated manufacturing systems; these being applied in respect of various Product Introduction, Manufacturing Planning and Control and Shop Floor Control scenarios (Weston 1993). Although important industrial benefits were realised the solutions were designed in an essentially bottom-up manner and as a result led naturally to application integration, rather than business integration. Hence to satisfy the requirements listed above (and hence achieve business integration) during the Model-Driven CIM project MSI research activity has sought to provide means of structuring and semiautomating the top-down specification, generation and configuration of the various software components of integrated manufacturing systems (Clements 1995). The approach is predicated on use of an integrating infrastructure (CIM-BIOSYS being the prime infrastructure used so far).

Essentially a three-staged approach has been taken towards this goal. In stage 1 a number of modelling methods and languages were selected and individually assessed in terms of their capability to model solutions in a way which can lead to model enactment ${ }^{1}$. Subsequently, stage 2 activity utilised MetaCASE Technology (i.e. a Computer Aided Software Engineering tool for building CASE tools) to extend and advance the use of existing methods and languages within four methods workbenches, see Figure 2.

Each workbench includes a model building and a model enactment capability and each has its own focus of application (in terms of the life-phase/modelling perspective matrix of GERAM) and strengths and weakness (as outlined in the following sections), this being inherited largely from its underlying modelling methodology and language. In the current stage 3 of the Model-Driven CIM project the four workbenches are being consolidated to realise wider, more effective and readily usable life-cycle engineering support.

\section{THE MODEL-DRIVEN CIM WORKBENCHES}

This section briefly describes each of the workbenches resulting from Model Driven CIM programme of work.

\subsection{Description of SEW-OSA}

SEW-OSA (Software Engineering Workbench based on CIM-OSA) combines CIM-OSA, Petri-Nets, Object-Oriented Design techniques and integration services of the CIM-BIOSYS infrastructure. It forms a major focus of research effort within the "Model-Driven CIM"

\footnotetext{
1. Model Enactment is a term used by MSI researchers to denote the process of turning models of solutions into physical elements of a working system. Typically using Model-Driven CIM workbenches the models created and derivatives of them are used to: (1) analyse alternative solutions (providing a basis for simulation); (2) to structure and semi-automate the creation of code (i.e. software components and integration software) used in the physical system; (3) to facilitate system implementation and test, via emulation (i.e. execution of partmodelled, part-real solutions); and (4) as a basis for co-ordinating and managing the runtime operation of the physical system in a model driven way
} 
Figure 2. Life-cycle Coverage of 4 Workbenches of Model Driven CIM

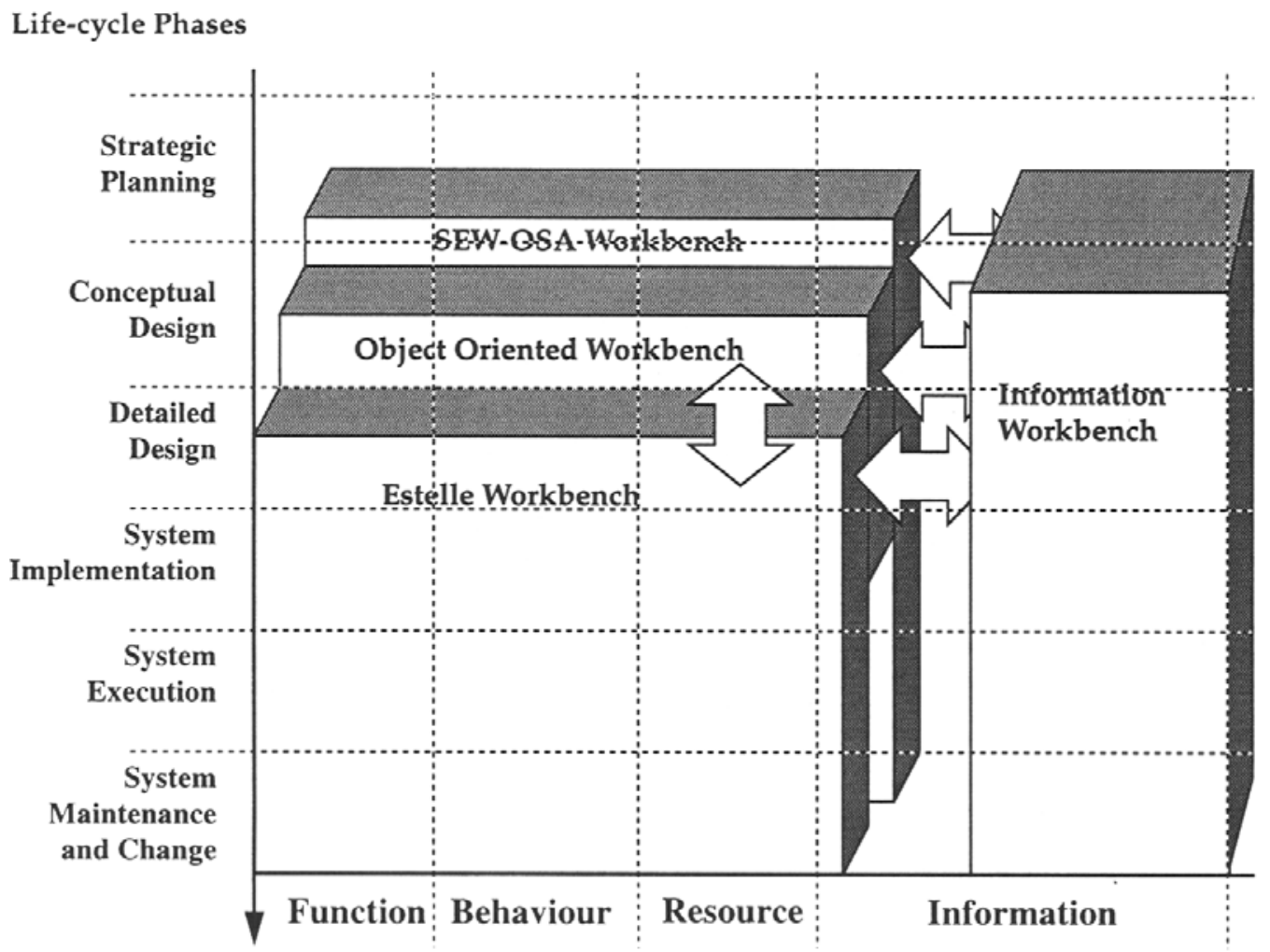

research programme and offers the framework of a toolset with a scope that embraces most phases of the IME life cycle (Aguiar 1994).

Thus, SEW-OSA provides an organised method for applying CIM-OSA to engineer lifecycle processes of integrated manufacturing systems by providing two classes of capability, namely: a model building capability and a model enactment capability. Each class of capability supports more than one modelling level of CIM-OSA whereby constructs are manipulated in the form of diagrams and templates within a CASE tool environment as a means of organising the design information. Essentially, the model building capability provides mechanisms and components which structure and support the specification and generation of consistent models which are are then used as input to the model enactment capability which provides mechanisms for either model simulation, emulation or execution.

Model building consist of two distinct stages of requirements definition and design specification. At the requirements definition stage, the workbench provides a set of diagrams that enable requirements to be described in a hierarchical manner, i.e. from the abstract definition of domains of interest (context and domain diagrams) to the more detailed specifications of individual enterprise activities (within structure, behaviour and functional 
diagrams).

At the design specification modelling level, the way in which functional entities (resources capable of carrying out enterprise activities) interact is determined and a specification of interaction requirements between the information system and the infrastructure is addressed using object and activity behaviour diagrams. Additionally, entity behaviour, resource and configuration diagrams are used to detail how the system resources should enact the desired system functionality. The workbench also provides a facility for describing resources within an object hierarchy.

Within the model enactment capability, two levels of enactment are possible. Firstly the generated models may be analysed with respect to appropriate performance criteria, this through generating stochastic timed petri-nets from the functional model using them to simulate the system behaviour. Secondly the system may be run with emulated components with system control governed by the elements of a business entity. Further migration of the system towards complete implementation can be accomplished by replacing emulated resources with real system components (i.e. machines, application programs etc.).

The runtime environment within the workbench includes the business entity to control and co-ordinate the execution of the system functionality through the interpretation of the models and a presentation entity for incorporation of actual resource components via the CIMBIOSYS integrating infrastructure (where the infrastructure provides distributed application transparency and configuration control).

Whilst the design to implementation approach is generally top-down, the workbench is intended to support an iterative design processes and where appropriate, provides a framework for updating system requirements and resources.

\subsection{Description of the Estelle workbench}

The Estelle workbench (ISO 1988) also provides support for much of the CIM system lifecycle through the application of a graphical design tool and various mechanisms for editing, debugging, simulating, emulating and operating a specified runtime environment. The emphasis with the Estelle workbench is that of co-ordinating the activities of distributed software functions through formally specifying their interaction. Thus designs specified using the tool consist of external descriptions of resources (including interfaces to hardware and software processes) which are co-ordinated in a manner which satisfies the system requirements. There is no distinct requirements definition stage as such and the designer is assigned responsibility to insuring that the system function is satisfied.

The Estelle workbench provides a graphical design tool which aids in the generation of the formal Estelle specification. This design tool is different from other available Estelle editors since it provides a system-wide view and is particularly suited to the description of manufacturing environments which are generally static in nature (i.e. manufacturing resources are generally not created or destroyed during system execution). The design tool aids in system visualisation and ensures that syntax rules and design rules of Estelle are upheld.

Estelle itself is highly object oriented, in that it provides a number of object classes for the description of systems (modules, interaction points, channels, messages). The behaviour of 
the modules is specified by state transition specifications whereby transitions are fired in response to receipt of messages. These object classes enable the co-ordination of distributed tasks in a system to be specified in a formal manner and hence subsequent processing of Estelle is possible. Estelle specifications can be tested and debugged using a number of available tools in the public domain. Such tools typically check for deadlock circumstances and unreachable states etc. Subsequently, a compiler and generator (adapted from a suite of tools developed at NIST (Sijelmassi 1991a, Sijelmassi 1991b)) are used to generate the interaction functionality of the distributed applications. In MSI's Estelle workbench, this functionality is combined with communication services of CIM-BIOSYS to enable distributed system execution and utilisation of distributed information retrieval and management services.

\subsection{Description of 00 Workbench}

The object-oriented CASE tool is based on the Booch methodology and provides an environment for the high level specification of systems through the application of object oriented techniques (Murgatroyd 1993, Booch 1992). Since the nature of contemporary manufacturing system is that of a collection of objects (i.e. machines, people, data etc.) communicating in a structured or semi-structured manner, the specification of manufacturing systems using object-oriented methods was highly appropriate. The workbench has a similar outlook to system design as that of the Estelle workbench, but with a broader scope, in that the workbench includes the specification of the information requirements of system objects and hence supports the creation of an idealised global information model.

Additionally the workbench enables the specification of objects at a deeper level than those of the Estelle workbench and provides a wider variety of co-ordination primitives. Object behaviour is captured using state transition diagrams and an additional interaction diagram can be used to check the sequential order of co-ordinating events.

Also within this tool, system resources may be represented in a resource class hierarchy, thus enabling creation of specific objects from the those of a more generic class. This capability reduces the effort required to create complex systems from scratch.

The workbench does not support an analysis phase as such, however the output from the object descriptions can be used to generate an equivalent Estelle specification which can be tested, emulated and executed in a similar manner to pure Estelle specifications.

\subsection{Information Modelling}

In their current form, none of the three functional workbenches incorporate a formal method of defining the information modelling requirements of the system. However, a separate CASE tool has been created at MSI which facilitates (a) the creation of EXPRESS-G models in a structured manner; (b) the generation of textural EXPRESS (ISO 1990) from these models and (c) access to the MSI's information modelling tools to automate the process of producing and populating database schema (Murgatroyd 1994). All three of the functional modelling approaches described in this paper are capable of producing a collection of information objects' which can be transferred to the information modelling environment in which the 
EXPRESS-G model and database schema can be constructed. The model produced in this way is 'ideal' and is termed the Global Information Schema. Elements in the global schema correspond with existing information resources, such as product information (which in the case study implementation are printed circuit boards), held in various file-based formats and databases. The information modelling environment also allows these information sources to be modelled in EXPRESS-G and hence elements of the global schema can be mapped onto elements of the existing information resources to enable a degree of automation in the generation of the information retrieval code (using for example LEX, YACC and proprietary functions in the case of files and SQL for databases). The way in which this retrieved data is formatted is also specified within the information modelling environment using BNF.

\section{CASE STUDY APPLICATION OF THE WORKBENCHES}

This section reports on an industrial case-study application of the MSI manufacturing system workbenches. The modelling domain chosen for this evaluation work comprised the Surface Mount Technology (SMT) assembly lines of a major UK supplier of electronics products. For the sake of conciseness, the model presented in this paper is a 'TO-BE' description of just one of these assembly lines. The model is a 'TO-BE' description in the sense that assumes the introduction of an integrating infrastructure for integration but it still reflects the current mode of operation of the assembly line. Figure 3 shows the basic arrangement and associated material and control flows for the SMT Assembly line. Thus, a batch of PCBs (the material) flow along the line and are inspected at two inspection points, i.e. after the printing process and whilst located on the inspection conveyor. The inspection operations are either triggered by problems detected by the operators or by a regular inspection which is programmed to occur (e.g. every " $n$ " PCBs manufactured at the point in question). Two buffers are used to smooth the flow of material and overcome imbalances in the line, thus avoiding inappropriate PCB queue sizes at the 'printed PCB buffer' and on the inspection conveyor.

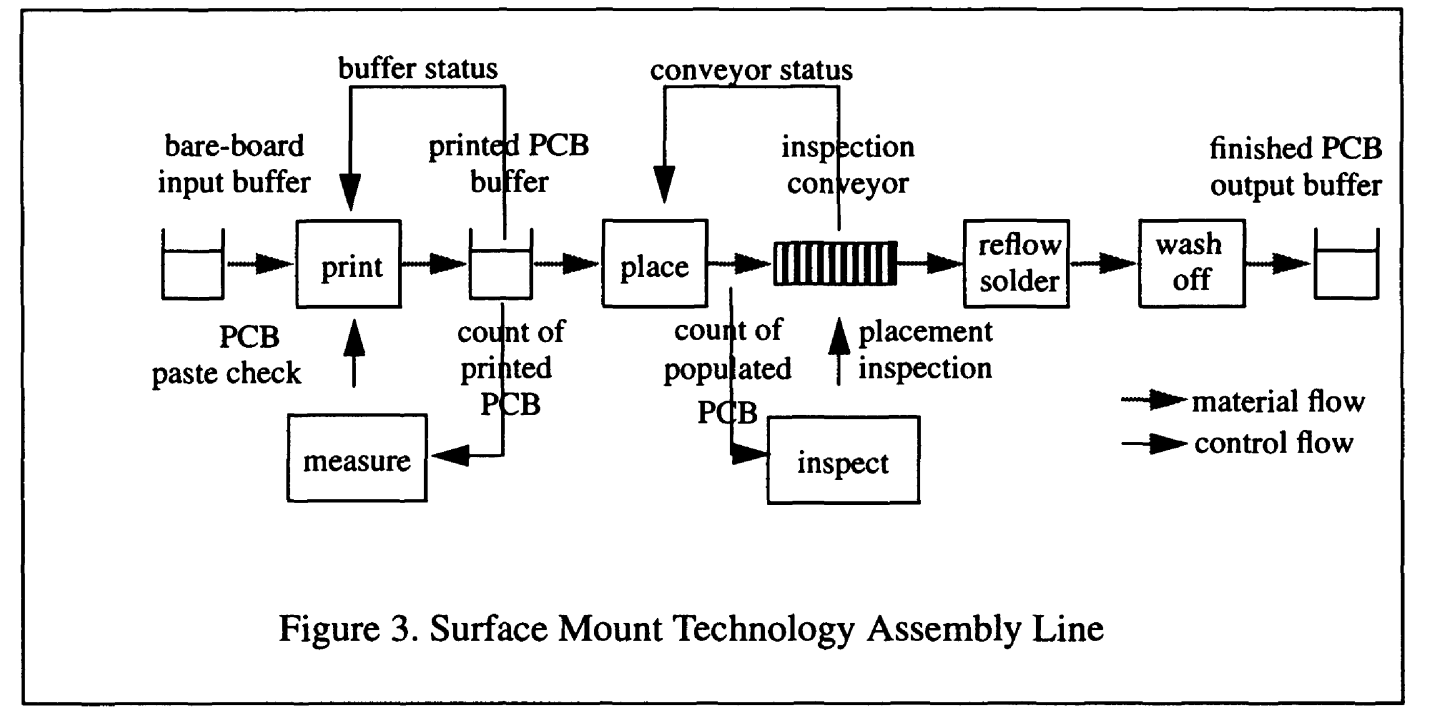

The demonstration system forms the basis for illustrating the use of the environments (workbenches) described in earlier sections of this paper to enable manufacturing system 
modelling and subsequent partially automated implementation and execution of processes over an integrating infrastructure. As an example of the modelling primitives of one of the workbenches, Figure 4 shows the object diagram within the Estelle workbench. Like the

Figure 4. Surface Mount Assembly Line as Viewed through Estelle Workbench Object Diagram

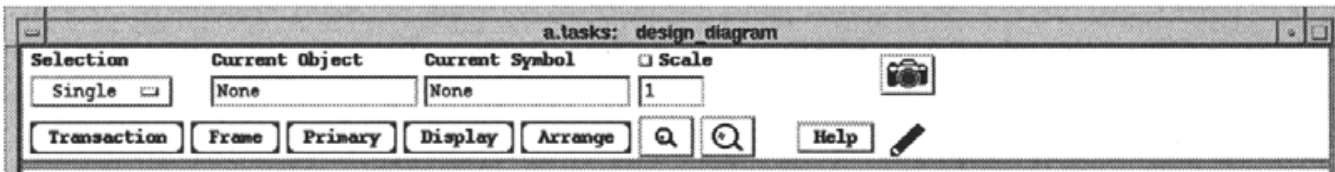

$\operatorname{sm}$

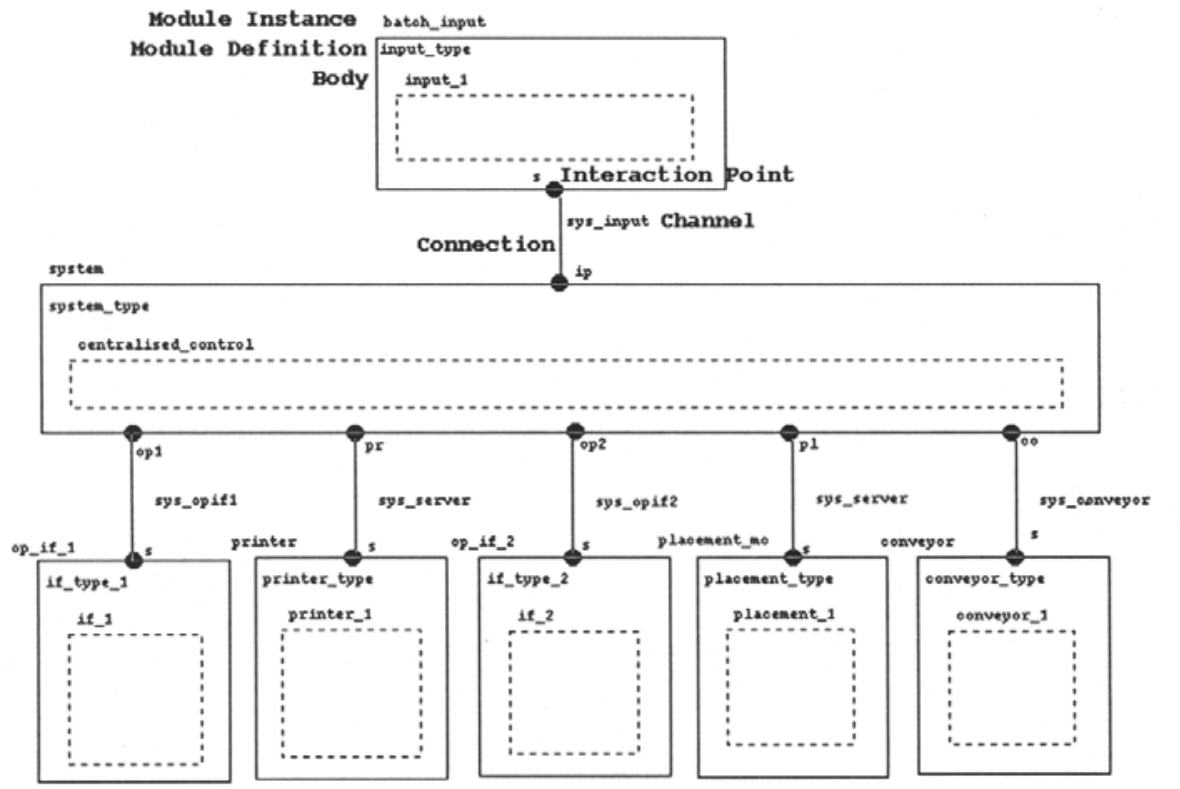

modelling environments of the other workbenches the design tool utilises the facilities of the IPSYS metaCASE tool for rapid CASE tool development. The diagram shows the individual Estelle modules identified from the case study example, i.e. physical machines (placement machine, printing machine, conveyor) and software processes (centralised controller, two interfaces to human operators and an external order input process). Communication between the modules is achieved through specific communication channels, each of which is associated with a particular set of messages. More details about the attributes of the objects such as channel definitions and internal module behaviour are specified through textual templates associated with each Estelle object and hence correct Estelle structure is maintained throughout the design process.

Figure 5 shows the schematic layout of the tools used to produce a running system to control the surface mount assembly line using the Estelle workbench. The interaction code for 
Figure 5. Schematic Layout of Estelle based workbench application.

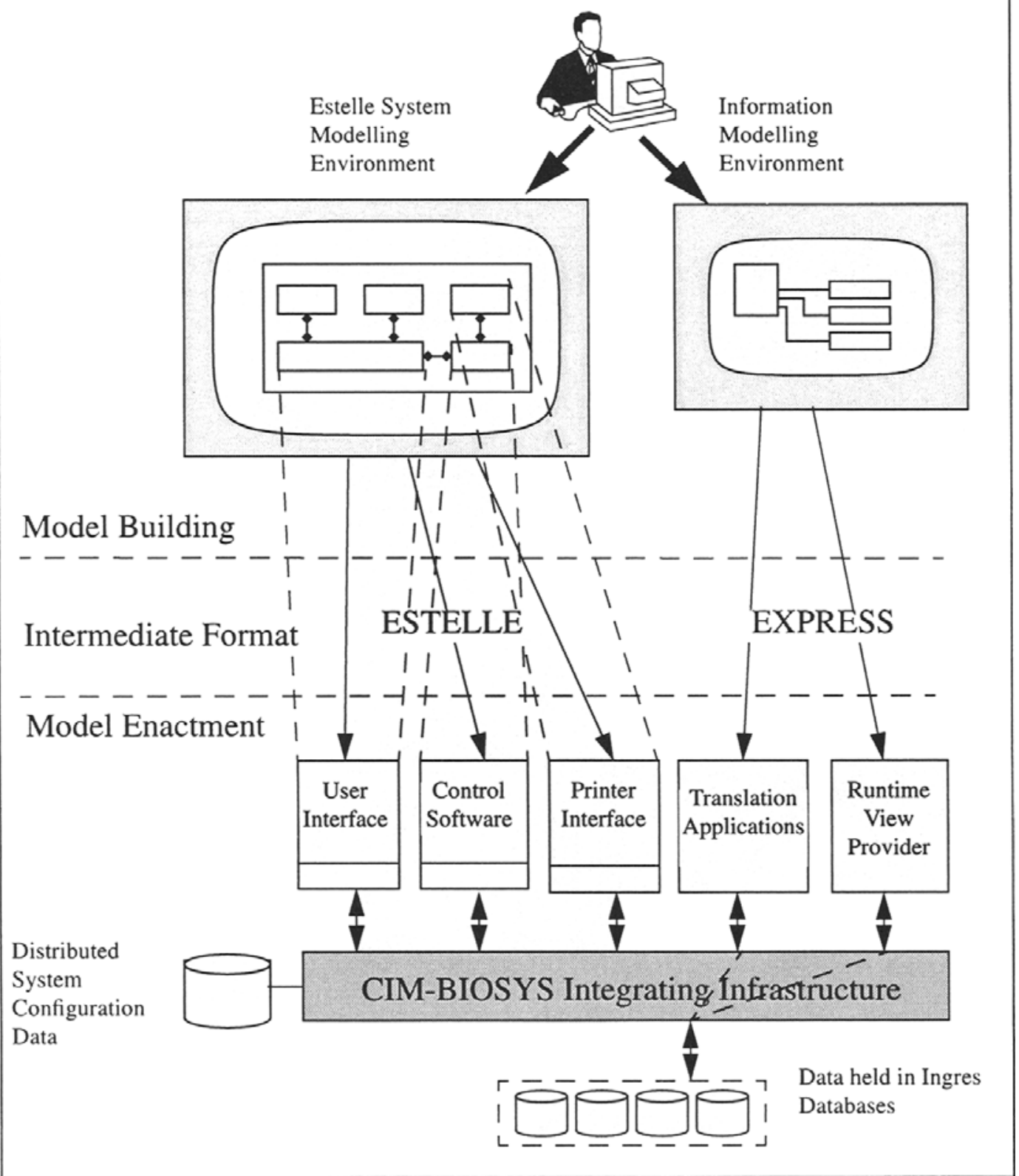

each distributed piece of software generated from the Estelle specification was combined with the communication libraries of CIM-BIOSYS and specific functions required by the module (such as graphical user interface definitions for the user interfaces and specific interfaces to machines).

Additionally the information requirements of the system modules were combined and captured in the information modelling workbench and this information was used as a basis for mapping simple information requests onto more complex information retrieval mechanisms required from specific databases using specific retrieval languages and standard data 
representation models. In our example scenario the user interfaces requested information concerning the positioning of a specific component on a printed circuit board from an Ingres database.

The absence of physical machines during the study work at the MSI prompted use of an animator to test and visualise the system execution. This animator runs as a Smalltalk application whereby each object within it (which represents a modelled object within the object-oriented workbench and derived from the Estelle based and SEW-OSA workbenches) receives messages from the distributed CIM-BIOSYS applications thereby animating its function.

Since the 'TO-BE' target system was modelled using the constructs supported by all three functional workbenches, one of the initial purposes of this study was to enable a critical comparison of the 3 functional modelling approaches. However, subsequent application of the methods revealed that particular constituent parts of each approach could be usefully applied within the other workbenches. Thus, in addition to a comparison of the methods, the study allowed a more complete and consolidated view to be extracted, although as the consolidated approach has yet to be implemented in a single toolset.

\section{CONSOLIDATED USE OF THE MODEL DRIVEN CIM WORKBENCHES}

Thus in creating and consolidating the Model-Driven CIM workbenches, MSI researchers have sought to define, enhance and unify a variety of modelling methods (and their underlying languages and graphical templates) and to provide means of making emerging enterprise modelling and enterprise integration techniques more usable and effective. Collectively the workbenches support much of the scope of GERAM and include a significant enhancement in known capability with respect to enterprise model enactment, i.e. structuring and semiautomating detailed design, implementation, build and operation phases of the GERAM lifecycle.

Currently and collectively Model-Driven CIM workbenches support the use of appropriate modelling technology for the shaded elements of the GERAM life-phase/ modelling perspective matrix, as depicted in Figure 6. Also within and across the workbenches a unification of modelling languages and methods has been achieved, this providing a means of mapping between each of the shaded elements of that matrix. Indeed MSI continues to evaluate enterprise engineering methodologies and modelling methodologies, this with respect to defining a consistent set of underlying modelling constructs and ontologies which can cover the complete GERAM matrix. The research has already demonstrated that very significant benefit can be realised by establishing synergistic relationships between complementary aspects of modelling methodologies and important first steps have been taken towards effectively mapping business goals onto appropriate enterprise activities in a model-driven (and hence highly flexible) manner. 
Figure 6. Areas of GERAM Matrix Supported by the "Model Driven CIM" Workbenches

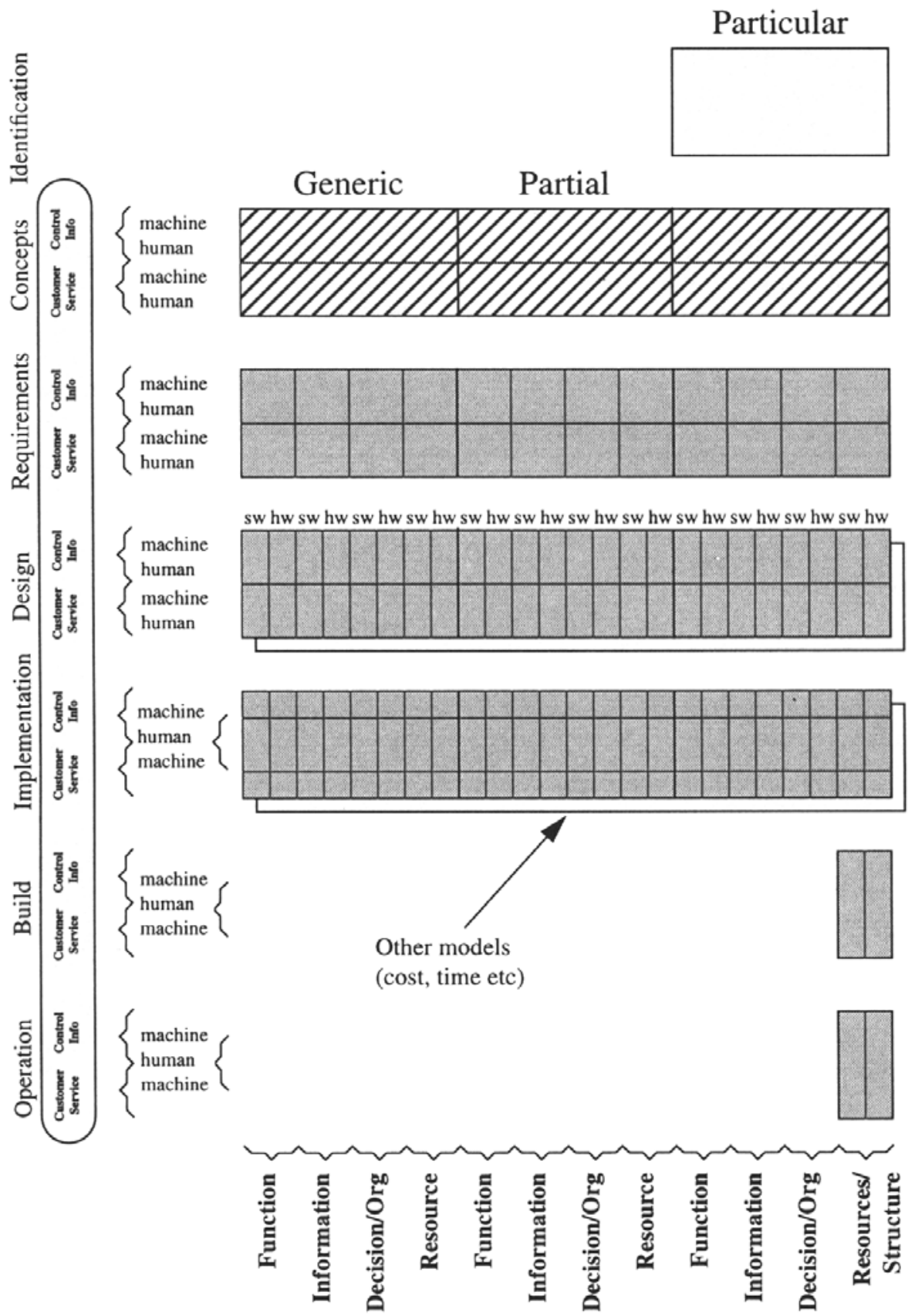

Area supported by the "Model Driven CIM" workbenches Area partly supported by the "Model Driven CIM" workbenches 


\section{CONCLUSION AND OBSERVATIONS}

This paper has provided an overview of the Model-Driven CIM project and its methods workbenches. It has also positioned the research with respect to the work of the IFAC/IFIP Task Force and the GERAM life- phase/modelling perspective matrix. A more detailed description of the Estelle workbench has illustrated model building and model enactment capabilities which are geared to supporting the "detailed design" life-phase through the "operation" life-phase of integrated systems which comprise concurrently operating and distributed manufacturing objects. This has also emphasised the importance of enacting models, i.e. to realise much greater benefit than that achievable by simply using models to support the conceptual design of solutions.

Although the Model-Driven CIM workbenches promise to produce solutions more effectively and rapidly for various types of manufacturing enterprise, before such benefits can be widely and more readily realised it has become clear that further research is required to:

(i) more formally unify modelling constructs and ontologies, so that enterprise integration technology can be placed on a more scientific footing;

(ii) select, advance and consolidate various means of supporting strategic planning and enterprise integration project planning activities (which correspond to GERAM Identification and Concepts life-phases), thereby more effectively bridging between business and technical worlds.

(iii) capture and develop families of enterprise models which provide guides and controls for the processes of realising, running and reconfiguring manufacturing enterprises.

The authors contend that only when enterprise models are available to support life-cycle engineering of enterprises will it generally be profitable for organisations to invest heavily in enterprise modelling. However, with the advent of methods workbenches which support the use of modelling technology it will become practical to model enterprise sufficiently completely and accurately for users to realise a major competitive edge. Clearly the chicken and the egg conundrum applies here, where good enterprise models will not be readily available until appropriate enterprise modelling technology can support their use, whereas achieving a wide acceptance of modelling technology relies to a large extent on the availability of appropriate reference models.

\section{ACKNOWLEDGEMENTS}

The authors would like to fully acknowledge the contributions made by other members of the Systems' Integration group within MSI, namely Marcos Aguiar, Shaun Murgatroyd, Paul Clements and Ian Coutts. Also the support of the ACME Directorate of EPSRC (and particularly Adrian Dent and Peter Gould) should be acknowledged. 


\section{REFERENCES}

Aguiar, M.W.C., Weston, R.H. (1994) SEW-OSA - Systems Engineering Workbench centred on CIM-OSA, Proc. of the 10th Int. Conf. on Computer Aided Manufacturing, Robotics and Factories of the Future CARS-FOF/94. Canada.

Bernus, P. and Nemes, L. (1995) A Framework to Define a Generic Enterprise Reference Architecture and Methodology (GERAM). Div. Rep. No. MTM 366 CSIRO Div of Manuf Tech, Preston, Victoria 3072 Australia.

Booch, G. (1992) Object Oriented Design with applications. Pub. Benjamin Cummins.

Clements, P., Coutts, IA., Murgatroyd, IS., Gilders, PG., Aguiar, MW., Weston, RH., Edwards, JE. and Hodgson, A. (1995) Model Driven CIM: A framework and toolset for the design of CIM Systems. Final Report, CDP Directorate, EPSRC, North Star Ave, Swindon, UK.

Fox, M.S. (1992) The TOVE Project, towards a common sense model of the Enterprise. Enterprise Integration, C. Petrie (Ed), Cambridge, MA, MIT Press.

ISO (1988) ISO 9074 Estelle: A Formal Description Technique Based on an Extended State Transition Model.

ISO (1990) ISO CD 10303-11: Exchange of Product Model-Data 11: The EXPRESS Language, Doc. N496 of ISO TC 184/SC 4/WG 1, July 90.

Murgatroyd, I.S., Edwards, J.M. and Weston R.H. (1993) Tools to Support the Design of Integrated Manufacturing Systems - An Object Oriented Approach - IEPM, June 93, Mons, Belguim.

Murgatroyd, I.S., Clements, P.E., Roberts, S., Sumpter, C. and Weston, R.H. (1994) An Information Modelling Methodology Applied to a Manufacturing Environment. NCMR '94. 10th National Conf. on Manufacturing Research, 13-15 Sept 94, Loughborough. Proc.: Advances in Manufacturing Technology VIII. pp335-339. ISBN 0-7484-0254-3.

Sijelmassi, R., Strausser, B. (1991a) The Portable Estelle Translator: an overview and user guide. NIST Technical Report.

Sijelmassi, R., Strausser, B. (1991b) The Distributed Implementation Generator: An overview and users Guide. NIST Technical Report.

Vallespur, B., Chen, D., Zanettin, M and Doumeingts, G. (1991) Definition of a CIM Architecture within the ESPRIT Project 'IMPACS'. Computer Applications in Production Engineering: Integration Aspects, G. Doumeingts, J. Browne, M. Tomljaprovich (Eds), Elsevier Amsterdam pp731-738.

Vernadat I, (1992) CIM-OSA - A European development for Enterprise Integration. Part2: Enterprise Modelling. Enterprise Integration Modelling, C. Petrie (Ed). The MIT Press, Cambridge, MA, pp189-204.

Weston, R.H. (1993) Steps Towards Enterprise-Wide Integration: a Definition of Need and First Generation Open Solutions, Int. J. Prod. Res.,Vol. 31, No.9, pp 2235-2254.

Williams, T.J. (1992) The Purdue Enterprise Reference Architecture., Instrument Society of America, Research Triangle Park, NC . 


\section{BIOGRAPHY}

Professor Richard Weston is the Head of the Manufacturing Systems Integration (MSI) Research Institute which comprises over 30 research associates and $\mathrm{PhD}$ students. He has over fifteen years experience of research in areas of machine control and systems integration and has supervised over fifty RAs and PhD students during that time. Richard is the author (or joint author) of over 250 refereed journal and conference publications in the field. He has been Principal Investigator for many UK research council grants which have generated output used by a variety of companies and institutions around the globe. He is a member of various national (EPSRC, DTI, LINK and BS) and international (ISO, IFAC, IFIP) committies serving the area and member of the board of five journals. Additionally, since 1993 he has been a Pro-Vice-Chancellor (Research) at Loughborough University.

Paul Gilders has been a researcher within the MSI Research Institute for 5 years subsequent to gaining his degree in Manufacturing Engineering and Management at Loughborough University. During this period his focus of research has been the implementation and modelling of distributed manufacturing systems. He also spent over two years working at the National Institute of Standards and Technology (NIST) in Gaithersburg, USA applying MSI's CIM-BIOSYS integrating infrastructure to NIST's manufacturing systems. Since 1993, Paul has been the prime developer of the Estelle workbench as part of the Model Driven CIM grant and is experienced in a number of function-based modelling methods and tools for manufacturing and machine control. He is currently involved with the integration of business issues within enterprise modelling and the development of reference models for manufacturing. 